\title{
PROFESORADO O LICENCIATURA Y OTRAS BIFURCACIONES EN LA ENSEÑANZA DE LA FILOSOFÍA
}

\author{
Elena Teresa José(1)
}

RESUMEN: Se plantea la bifurcación de los planes de estudio de Filosofía en profesorado o licenciatura, la que responde a la vez a otra división: enseñanza o investigación. Se formulan las siguientes preguntas: ¿enseñar implicar investigar? ¿una investigación debería estar expresada de manera comprensible siguiendo criterios didácticos? ¿habría una metodología de la investigación escindida de una metodología de la enseñanza y viceversa? ¿es posible enseñar Filosofía o sólo es dable enseñar a filosofar? Se da cuenta de la etimología de las palabras "enseñanza" e "investigación", observando los rasgos comunes de ambas y se aboga por una enseñanza que implique investigación y una investigación que se exprese en forma clara e inteligible. Respecto a los métodos de investigación y de enseñanza, se estima que la metodología de la investigación y la metodología de la enseñanza en Filosofía, no son cosas extrañas ni distantes. Ante la dicotomía: "Enseñar filosofía" o "enseñar a filosofar" se considera que si la entendemos en función de contenido y método, ambas se compatibilizan, pero como dos posturas ante la verdad filosófica, son incompatibles. Por último se propone la unificación de los planes de estudio.

\section{Planteo del problema.}

En primer lugar hay que aclarar que este trabajo, en lo que a normativas y tradición se refiere, está contextualizado en lo que sucede en mi país: Argentina.

La ramificación entre profesorado o licenciatura en Filosofía en los planes de estudio responde a la vez a otra división: enseñanza o investigación, ya que la principal incumbencia que habilita a un profesor es enseñar y a un licenciado, investigar. Así tenemos el caso de que un profesor recibido en un terciario (ahora educación superior no universitaria) tiene ante la Junta de Clasificaciones más puntaje para enseñar en el secundario (ahora Polimodal), por la especificidad del título, que un Doctor en la misma disciplina, cuyo título de grado es licenciado, por ejemplo.

Esto no está mal, porque el profesor tiene una formación docente y estudió para eso: enseñar. Pero esto no es tan simple, ya que dejamos al Licenciado sin incumbencias para enseñar (en la EGB 3, Polimodal y Superior No-Universitaria) y al Profesor sin incumbencias para investigar.

Los planes de estudio contemplan por lo general, un tronco común de materias básicas y luego se abren dos ramas. Los que siguen la licenciatura deberán tomar seminarios o materias más específicas, que pueden ser orientadas hacia una 
especialización (Ética, Epistemología, Metafísica, etc.) y otras que son de tipo procedimental, como Metodología de la Investigación, y esto culmina con una tesis de grado, que también se ha dado en llamar tesina. Los que siguen el profesorado deben tomar generalmente materias como Psicología; Sistema Educativo; Institución Escolar; Didáctica General y Didáctica Especial.

Ahora bien, quien aspira a enseñar en los claustros universitarios sabe muy bien que su título de licenciado lo habilita para ello, y hasta algunos jurados lo consideran con más rango que el de profesor. Pero quien tiene claro que sus posibilidades laborales están en el nivel medio, se inclina por el profesorado.

La reforma educativa argentina trajo aparejada muchos, muchísimos documentos, entre los cuales hay una resolución ministerial nacional que fija que se considera de grado a las carreras universitarias que tienen por lo menos 2600 horas. Para los profesorados se estipula un mínimo de 2800 horas, por lo que la exigencia para un profesorado resulta con más carga horaria que una licenciatura. En consecuencia, en los nuevos planes lo más común es que quien opte por el profesorado, recorra casi el mismo plan que el licenciado (menos la tesis) y deba tomar el bloque de materias de formación docente.

A la hora de discutir los planes de estudio y los alcances de los títulos, nos preguntamos cuáles son las competencias que deberán tener los graduados de una u otra opción.

Un profesional de Filosofía, Historia, Química, Matemática, Biología, Física o cualquier disciplina debe ante todo, saber de lo que su disciplina trata y además estar actualizado.

Y aquí se me ocurre formular cuatro preguntas básicas que valen para todos los niveles del sistema educativo.

1. ¿enseñar implicar investigar?

2. ¿una investigación debería estar expresada de manera comprensible siguiendo criterios didácticos?

3. ¿habría una metodología de la investigación escindida de una metodología de la enseñanza y viceversa?

4. ¿es posible enseñar Filosofía o solo es dable enseñar a filosofar?

\section{Enseñanza e investigación}

Con respecto a la pregunta 1, resulta difícil dar una definición de enseñar, ya que, por una parte, hay diversas concepciones acerca de la enseñanza y por otra, como dice Borges: 
Cometemos un error muy común cuando creemos ignorar algo porque somos incapaces de definirlo. Si estuviéramos de un humor chestertoniano, diríamos que sólo podemos definir algo cuando no sabemos nada de ello". (Borges, 2001:33/34).

No obstante, ensayaremos algunas aproximaciones acerca de lo que es "enseñar", a partir del capítulo 6 del libro de Walter Kohan, titulado Infancia: Entre educación y Filosofía, quien dice:

¿qué significa enseñar? No sabemos la respuesta.

[...] Ciertamente, estamos ante una pregunta compleja, abierta, polémica, con una larga historia de enunciaciones y pretendidas soluciones. No la trataremos comprensivamente. No la agotaremos. La recrearemos.

[...] Vamos a visitar la etimología. "Enseñar" forma parte de un grupo de palabras del mismo grupo semántico de "educar", al igual que otros términos como "instruir" o "formar". Todos estos términos se originan en el latín y comparten una cierta idea semejante a educar: la de darle algo a alguien que no lo posee. "Enseñar" viene de insignare, textualmente "dar o poner un signo", "dar un ejemplo".La base del término es la raíz indoeuropea sekw, con el significado de "seguir". Signum, el elemento principal de insignare remite al sentido de "signo", "señal", "marca" que se sigue para alcanzar algo. El "signo" es "lo que se sigue". De modo que lo que se da en el enseñar es un signo, una señal a ser descifrada.

Y más adelante agrega:

[...] Como su etimología lo sugiere, enseñar debe tener que ver con propiciar signos, señales, marcas que pueden ser seguidas. (Kohan, 2003 a: 183, 185, 188 El autor aclara que la etimología de "enseñar" fue extraída de A. Castello, C. Marcico. "Glosario etimológico de términos usuales en la praxis docente. 1988, p. 15)

Veamos la etimología de investigar:

"Investigar" proviene del latín (MACCHI, Luis. Diccionario de la lengua latina $<1948>$. Bs. As., SEI). investigare "seguir la pista, la huella". A su vez, de la palabra vestigium, pista (literalmente "dirigirse hacia la pista o huella"). (2)

Observemos que las huellas son signos, los vestigios son signos.

Ambos términos, "enseñar" e "investigar" tienen que ver con huellas, signos, marcas.

Si investigar es producir nuevas ideas, las ideas -que en una investigación se expresan en lenguaje- son signos (escritura) producto de una interpretación. Así el psicólogo interpreta el dibujo para realizar el diagnóstico; el químico interpreta el color del papel que introduce en la sustancia; el arqueólogo la forma, cocción y 
dibujo de la cerámica; el médico las manchas de la piel; el sociólogo el hecho de la huelga o el texto que habla sobre este hecho.

Recordemos que para que haya proceso semiósico, deben darse al menos tres elementos: el signo o vehículo señal, el designado y el intérprete.

Por ejemplo, si las velas rojas, negras o blancas nos informan acerca de su color rojo, negro, blanco y nada más, no son signo, no hay proceso semiósico.

Pero cuando Teseo, el célebre héroe griego, al partir de viaje, se puso de acuerdo con su padre el rey Egeo en que las velas negras en su barco, significarían desgracia, y las blancas éxito, ésta simplísima señalización ya fue un sistema de signos; el color de las velas no sólo informaban de su color, sino además algo que estaba fuera de él; el color era un SIGNO. Las velas negras significaban para Egeo la muerte de su hijo, para los marinos de los siglos XVI-XVII, un barco pirata" (Kondrátov, 1973: 14/15).

Entonces, en una investigación hay procesos semiósicos, ya que no hay investigación sin interpretación.

N. Rusell Hanson trabaja con el siguiente ejemplo:

Pensemos en Johanes Kepler: imaginémoslo en una colina mirando el amanecer. Con él está Tycho Brahe. Pero Tycho, siguiendo a Ptolomeo y a Aristóteles, al menos en esto, sostiene que la tierra está fija y que los demás cuerpos celestes se mueven alrededor de ella. ¿Ven Kepler y Tycho la misma cosa en el Este del amanecer? (Hanson: 1977: 13)

La respuesta a esta pregunta será que los dos reciben los mismos datos sensoriales del mismo fenómeno, pero Copérnico fue capaz de interpretar el paisaje (signo) de manera diferente a Ptolomeo y cambió el paradigma de la Física, produjo nuevas ideas.

Efectivamente, si Tycho y Kepler hubieran poseído cada uno una cámara fotográfica, el ojo de la máquina hubiese registrado la misma cosa. Pero el "corpus científico" no se constituye con el mero registro y acumulación de datos, sino que a esos datos hay que interpretarlos; Tycho y Kepler ven el mismo paisaje, pero uno lo interpreta desde una teoría ptolemaica y otro desde una copernicana.

Y si investigar tiene que ver con la interpretación de signos y enseñar con "propiciar signos", sería difícil pensar en una enseñanza esquematizada, repetitiva, clonada, reproducida, sin que interprete y se deje interpretar, comprender, producir, ensayar, intentar, transformar, que tiene que ver con la creatividad en la enseñanza y en la investigación y también tiene que ver con otra dicotomía clásica: "enseñar Filosofía" o "enseñar a filosofar", la que será tratada en el apartado 4.

El maestro, profesor, enseñante o como se llame, no es un cerebro lleno de información o contenido, sino una persona que tiene internalizadas una serie de 
valores e ideas propias que pueden ir desde las más originales hasta una elaboración personal de las lecturas que selecciona. Si uno pide a los estudiantes integración propia de conocimientos, no concibo profesor que sea mero "repetidor". Y para no repetir es necesario originar ideas, es decir investigar, por más modesta que esta investigación sea.

La informática nos brinda programas de autoaprendizaje que realmente maravillan, pero que sólo difunden contenidos, corrigen, indican. La presencia del profesor o maestro en la educación, me parece muy impotante.

Observemos que Jaques Rancière en su libro "El maestro ignorante" argumenta en contra de la explicación. Su personaje, Jean Joseph Jacotot, un maestro desterrado, no se puede comunicar con sus alumnos, ya que éstos hablan otro idioma que él no conoce y sin embargo, aprenden sin sus explicaciones, pero con su presencia de maestro que "da señales".

Me simpatiza un tipo de enseñanza personalizada, presencial, y que asimismo contemple la producción intelectual del maestro, su propio pensamiento. Me resulta difícil pensar que alguien pueda repetir contenidos sin haberlos seleccionado, interpretado, apropiado, elaborado, adaptado para ese grupo de alumnos, integrado con otros conocimientos o experiencias anteriores; sin haberles dado una coloración personal, y aunque estas últimas operaciones pertenecen al campo de la didáctica, el docente debe tener criterios investigativos para llevarlas a cabo. Las investigaciones no son sólo disciplinares, sino también hay una investigación - acción, que permite innovar en el aula. Docencia e investigación se retroalimentan.

Al respecto, Guillermo Obiols, quien apoya sus ideas en el filósofo español José Gaos y en el italiano Rodolfo Mondolfo, afirma que:

"Tradicionalmente las universidades nacionales en las facultades de ciencias exactas y naturales y en las de humanidades han organizado estudios de licenciatura. El título 'licenciado" ha significado el reconocimiento a quien lo obtiene de una aptitud para entender en los asuntos de que se trate: matemática, biología, historia o filosofía. Este 'entender' supone que se reconoce que el licenciado ha cultivado una disciplina o un área de estudios y que es capaz de algún tipo de producción en su campo, tal es el sentido de la tesis de licenciatura, trabajo que el licenciado ha producido con la guía de sus profesores, que es el primer trabajo de una cierta envergadura, que lo habilita y que se espera que no sea el último. Un plan de estudios de licenciatura debería facilitar que el estudiante aprendiera a realizar este tipo de producción en su disciplina. El plan no sólo debería garantizar que el estudiante conozca la historia de su disciplina, los desarrollos actuales de la misma, sus distintas teorías, los puntos sobre los cuales puede haber acuerdo y aquellos en los que hay discrepancias, sino que debería apuntar a que el graduado pueda realizar algún tipo de producción más o menos original en la misma".

Y agrega: 
En general, se reconoce en los estudios de filosofía en las universidades más prestigiosas del mundo que la función de los profesores no es tanto la de proporcionar una información general filosófica, que pueda hallarse en diferentes libros, como la de iniciar en una práctica de trabajo filosófico mostrando en cursos y seminarios los propios trabajos realizados por los profesores". (Obiols, 1997)

Lo que afirma Obiols como la función de los profesores universitarios, me parece que se puede extender a los otros niveles de enseñanza, donde el Profesor debería también generar con sus investigaciones un material bibliográfico (o la adaptación del mismo) de acuerdo a la institución, los estudiantes, los intereses, las circunstancias, y los temas de actualidad que preocupan, para hacer de la enseñanza una experiencia renovadora e innovadora y no la mera transmisión de esas "verdades en la que cómodamente estamos instalados". Todo esto no se improvisa. Necesita de docentes transformadores, críticos y creativos.

La investigación de nuestras propias prácticas docentes, es también investigación. Y si bien la investigación en química es disciplinar y en la enseñanza de la química es educativa, en Filosofía esto no es tan así, ya que:

[...] la didáctica de la filosofía es esencial y constitutivamente filosófica y no un mero instrumento técnico externo de apoyo a la misma actividad filosófica. (Cienfuentes, 1997: 87).

\section{Criterios didácticos y técnicas expositivas.}

Con relación a la pregunta 2 , sabemos que no hay ciencia de lo privado, que un cuerpo de conocimiento es científico cuando es puesto a consideración de nuestros pares, lectores, estudiantes, otros investigadores, otros puntos de vista.

Casualmente una de las acepciones de "enseñar" es "mostrar".

Es por eso que las reuniones científicas (Congresos, Jornadas, Coloquios, etc.), las publicaciones, son condición necesaria para la investigación. Y vaya si no se necesitan criterios didácticos para escribir una comunicación, para discutir una tesis o para publicar un artículo.

Dice Jaspers:

Hay que aceptar la exigencia de que la filosofía sea accesible a todo el mundo. Los prolijos caminos de la filosofía que recogen los profesionales de ella, sólo tienen realmente sentido si desembocan en el hombre. (Jaspers, 1970: 8)

Cuando se propician signos para que otros interpreten, para que sirvan de huellas a fin de transitar nuevos senderos en cuyo tránsito podamos pensar ideas diferentes, es necesaria la claridad conceptual, lo que no implica que deje de usarse un lenguaje metafórico, donante de sentido, sino todo lo contrario.

Existe en algunos círculos, la creencia de que quien habla en "difícil" tiene más nivel y quien se expresa con claridad es más elemental. Me parece que esto es un 
craso error y que, casualmente, quien habla con claridad, es porque ha alcanzado a elaborar sus ideas de modo más inteligible.

Hay una conocida frase hegeliana según la cual un gran hombre condena a los demás a tener que interpretarle.

Sin embargo, cuando Hegel -a pesar suyo porque siempre aspira a enseñar en la Universidad- tiene que desarrollar su actividad profesional en el Gimnasio, en Nurenberg, le escribe a su amigo y protector I. Niethammer, diciendo:

es más fácil hacerse incomprensible de una forma sublime, que ser comprensible de una forma sencilla, y que, la instrucción de la juventud y la preparación de la materia para ello constituyen la última piedra de toque de la claridad. (Hegel, 2000:20).

\section{Al respecto dice Arsenio Ginzo en la Introducción a los Escritos Pedagógicos}

Este "descenso" al mundo del Gimnasio va a tener para Hegel al menos la ventaja de una mayor claridad, fluidez y libertad expositivas, obligándole a reconsiderar su mundo filosófico en función de sus nuevos destinatarios. Hegel no duda en reconocer esa dimensión positiva de su nueva actividad, considerando que constituye el medio más eficaz para conseguir la deseada soltura expositiva. A este respecto no tiene reparo en contrastar la libertad expositiva conseguida, con la oscuridad o la servidumbre respecto a la letra, que le aquejaban durante el período de Jena. (Hegel, 2000:24).

Y esto me da lugar para hablar de otras famosas divisiones en la enseñanza: Educación General Básica o Primaria, Polimodal o Secundaria, Superior no universitaria o Terciaria y Universidad (Grado y Postgrado).

No estoy en contra de esas divisiones, me parecen necesarias y constitutivas del sistema educativo, pero me pregunto, por ejemplo: ¿cuál es la diferencia entre dar una clase universitaria en Filosofía en el Grado o en el Postgrado? Se supone que las exigencias respecto de un trabajo para evaluar, son mayores en el Postgrado, pero a los fines de la enseñanza y aún de la Bibliografía chabría una diferencia sustancial entre estos dos niveles, máxime teniendo en cuenta que los títulos de Postgrado no otorgan incumbencias, sino que sólo son académicos y no profesionales, y entonces en un Postgrado de Filosofía puede inscribirse un profesional de cualquier disciplina? Estimo que la diferencia es de tipo gradual y no saltos de tipo cualitativos.

¿Cuál es -a los fines prácticos- la diferencia entre los cursos de "Actualización", "Capacitación" y de "Perfeccionamiento", si tienen la misma carga horaria y la misma evaluación? ¿La diferencia es sólo a los fines del puntaje que otorga? Si ello es así resultan falsas dicotomías.

Nuestra Ley de Educación Superior No 24.521, que data de 1995, en su artículo $7^{\circ}$ estipula que podrán ingresar a la Universidad, como excepción, los mayores de 25 
años que no tengan título secundario, siempre que demuestren que tienen preparación y/o experiencia laboral acorde con los estudios que se proponen iniciar, así como aptitudes y conocimientos suficientes para cursarlos satisfactoriamente. Las pruebas de evaluación están a cargo de cada universidad, de acuerdo a los criterios que en cada caso establezcan.

Este artículo ha sido muy polémico y las Universidades no estuvieron de acuerdo en su aplicación.

Recientemente, en el año 2003, se ha promulgado la Ley $N^{\circ} 25.754$ que modifica el artículo 39 de la Ley de Educación Superior. El mismo establece que podrán acceder a la formación de postgrado, el postulante del nivel superior no universitario (o sea terciario) de cuatro (4) años de duración como mínimo, siempre que reúna los prerrequisitos que determine el Comité Académico o la autoridad equivalente, a fin de comprobar que su formación resulte compatible con las exigencias del postgrado al que aspira. También estipula que la admisión y la obtención del título de postgrado no acredita de manera alguna el título de grado anterior correspondiente al mismo.

La aplicación de esta Ley es muy resistida también en muchísimas Universidades, entre ellas la Institución universitaria a la que pertenezco, que no la reglamentó porque está en desacuerdo con la normativa.

Estos ejemplos son para argumentar que a veces reparamos más en las divisiones, como compartimentos estancos, que en la realidad, que es en última instancia la que nos demostrará la operatividad o no de la medida.

En definitiva, creo que docencia e investigación, aunque distintas, se retroalimentan; que el docente debe investigar; que el investigador debe tener criterios didácticos. La etimología de los términos "enseñar" e "investigar" también ha dado cuenta de esto. Las falsas dicotomías obstruyen la apertura y flexibilidad deseables en materia educativa y no pocas veces la rigidez de las estructuras obstaculiza el acto pedagógico, que es lo genuinamente sustantivo.

\section{Métodos de investigación y métodos de enseñanza.}

En lo que hace a la pregunta 3, mucho se ha hablado de tácticas (generales) como método y estrategias (particulares) como técnicas para investigar. Cada disciplina tiene métodos y técnicas propias, pero a los resultados de la investigación se los debe hacer conocer -como se ha dicho- de la manera más inteligible posible y es aquí donde el investigador (por lo menos en Filosofía) aunque no esté en un aula, hace docencia. Demás está decir que se puede hacer docencia en el laboratorio, en la calle, en la fábrica, en la oficina, en un foro de discusión, en un comité de pares entre otros.

Pero el caso de la Filosofía que nos ocupa es muy especial, ya que las ideas que se sostienen acerca de la enseñanza de la Filosofía, guardan una íntima relación con la concepción de Filosofía que se abrace. 
La metodología de la investigación y la metodología de la enseñanza en Filosofía no son cosas extrañas ni distantes y tanto el licenciado como el profesor deberían manejarlas.

Tampoco estoy diciendo que la metodología de la investigación en filosofía es exactamente la misma cosa que la del aprendizaje, pero creo que están más fuertemente entrelazadas que en otras disciplinas.

Así, el filósofo peruano Augusto Salazar Bondy plantea como métodos didácticos los derivados de líneas de abordaje de la filosofía como el análisis filosófico y la fenomenología, por ejemplo.

Con respecto al análisis filosófico, dice:

Siendo el análisis del lenguaje un proceder normal en filosofía, no puede considerarse extraño a la metodología de la enseñanza filosófica. Las figuras más características del pensamiento han filosofado en trato directo con la problemática lingüística; su filosofar es en parte fruto de este quehacer y está impregnado de sus incitaciones y sus hallazgos. Siendo el acceso al filosofar el objetivo supremo de la didáctica filosófica, no podrá conseguir su propósito si prescinde del abordaje crítico del instrumento lingüístico. (Salazar Bondy, 1974:175).

En lo que hace a las líneas fenomenológica y crítica asevera:

Que la didáctica filosófica pueda aplicar un método fenomenológico y crítico es posible porque cabe filosofar fenomenológica y críticamente. (Salazar Bondy, 1974:175).

Al abordar la cuarta y última cuestión, nos preguntamos:

\section{Enseñar filosofía o enseñar a filosofar ¿̇con o inclusiva "o" exclusiva?}

Dentro de la temática de la enseñanza de la Filosofía, aparece recurrentemente el tema: enseñar filosofía (representada por el enfoque hegeliano) o enseñar a filosofar (representada con el enfoque kantiano)

Dice Kant en la Crítica de la Razón Pura:

Solamente puede aprenderse a filosofar, o sea a ejercitar el talento de la razón en la observancia de sus principios universales en ciertos intentos existentes, pero reservándose siempre el derecho de la razón a investigar esos principios en sus propias fuentes y confirmarlos o rechazarlos. (Kant, 1965: 401).

Y en otro texto:

No se debe enseñar pensamientos, sino enseñar a pensar; al alumno no hay que transportarlo, sino dirigirle si es que tenemos la intención de que en el futuro sea capaz de caminar por sí mismo. 
(...) permítaseme decir que se abusa de la confianza de la gente cuando en lugar de ampliar la capacidad de entendimiento de la juventud que se ha puesto en nuestras manos y formarla para que en el futuro pueda madurar la propia inteligencia, se la embauca en una filosofía clausurada y completa que ha sido elucubrada para ellos por otros.

(...) al autor filosófico que se pone de libro de texto, debe considerársele no como el modelo de juicio, sino sólo como ocasión para juzgar sobre él o, incluso, contra él. (Kant, 1965: 401)

Hay quienes interpretan que la expresión "enseñar filosofía" significa enseñar contenidos y que "enseñar a filosofar" hace referencia a procedimientos, por lo que la "o" de la dicotomía sería inclusiva y, en verdad, se trataría de un falso dilema, ya que son dos caras de una mismo moneda(3).

Pero creo que el planteo va más allá.

Voy a tomar una distinción de Foucault entre verdad y experiencia(4). Se puede escribir un libro (o enseñar, dar una clase, una conferencia, etc.) como verdad o como experiencia. Aunque toda experiencia supone la afirmación de cierta verdad, la experiencia se opone a la verdad y permite volverse sobre ella, transformarla. Así, en la experiencia, lo esencial no se encuentra en la constatación de una verdad histórica, sino que es única e irrepetible, no es una copia fiel, sino que es algo de la cual uno mismo sale transformado/a. En esta perspectiva, las dos posiciones parecen irreconciliables, o se escribe un libro como verdad, para transmitir una verdad que se sabe de antemano o se lo escribe como experiencia, para transformar la relación que se tiene con la verdad. En este caso, la "o" resulta ser exclusiva.

Entonces, aquí ya no se trata solamente de contenidos versus procedimientos, sino que la cuestión pasa por la actitud que se tenga ante la verdad filosófica y por el concepto mismo de Filosofía.

Creo que la Lógica es una ciencia independiente de la Filosofía y que la Filosofía es una actividad que debería desarrollarse partiendo de la base de que no hay verdades absolutas; también de que todo filósofo/a debe admitir, como inherente a su sistema de creencias filosóficas, la posibilidad de revisar permanentemente sus verdades.

Esto, de ninguna manera implica que se deban dejar de lado los contenidos, sino que lo que difiere fundamentalmente-repitámoslo una vez más- es la actitud respecto de la verdad.

Los contenidos y la preparación filosófica son insoslayables, pero la Filosofía es una manera, un modo de andar por sí mismo/a el camino y no un punto al cual se llega y en esto me permito discrepar con Hegel y acuerdo con Kant en que la Filosofía no puede ser clausurada. La ciencia tampoco. 
¿O acaso la Filosofía no es otra cosa que aprender a pensar por sí mismo/a? ¿O acaso aprender a pensar por sí mismo/a no es crear y re-crear dejando siempre posibilidades abiertas?

\section{Conclusiones}

1) Respecto al primer punto, una propuesta sería que se expidan títulos de Graduados Universitarios (o algo así) en Filosofía, que habiliten para docencia, investigación y otras funciones propias. Estimo que este criterio implicaría planes no bifurcados, que tiendan a una formación más integral de los profesionales.

Como no hay tradición en esto, traería aparejado algunos problemas según las pautas estándares actuales. Hubieron algunos planes de estudio de profesorado de filosofía en universidades nacionales del país en las décadas del 60 y 70 que contemplaban todas las materias de una licenciatura (algunos incluso Tesina), de 5 años más las materias pedagógicas que tuvieron obstáculos en algunas universidades del mismo país y del extranjero para acceder al doctorado, porque se consideró que para acceder a éste, era necesario el título de licenciado.

En el actual contexto, de acuerdo a las normativas vigente, no sería posible un título unificado, pero como en educación las reformas, los convenios y acuerdos son -o deberían ser- permanentes, el objeto de este artículo, es presentar mis argumentaciones como material de insumo para el intercambio de opiniones y análisis crítico del tema.

2) La unificación del profesorado y la licenciatura, traería aparejada la idea de no pensar tan dicotómicamente la actividad docencia - investigación.

3) Si acordamos que el modo de enseñar Filosofía que se tenga está asociada con el concepto de Filosofía que se sostenga, es decir si hay coherencia en ello, tampoco estaría tan escindido el método que usamos para enseñar con el que empleamos para investigar, ya que lo que desarrollamos en ambas instancias es la actividad de filosofar.

4) Si por "enseñar Filosofía" o "enseñar a filosofar" se entiende contenido versus procedimiento, deberíamos interpretar la "o" como inclusiva. Si se entienden como dos actitudes ante la verdad filosófica, la "o" debe interpretarse como exclusiva tanto en modo de entender lo que es la Filosofía, como en la forma de enseñarla.

En suma, pienso, citando a Walter Kohan, que (como enseñanza o como investigación).

Entendemos la práctica de la filosofía como una experiencia intersubjetiva del pensar. La experiencia, no la técnica, está en la base de un pensar tal. Cuando se busca reproducir o generalizar una experiencia, ésta se vuelve un experimento, espejo de lo mismo. Al contrario, la filosofía, como experiencia, no puede normalizarse, uniformizarse, estandardizarse. Nadie puede hacer experiencias por 
otro, nadie puede pensar por otro, nadie puede filosofar por otro. (Kohan, 2003 a: 108)

[...] se trata, al fin, de un ejercicio. Pensamiento vivo y en acto. Nada de esquemas, clasificaciones, generalizaciones. Filosofía en acto, gesto de interrogación, irrenunciable, sobre la propia práctica. Experiencia singular que da lugar a un pensamiento singular. (Kohan, 2003 b: 74).

[...] Esta es la tarea de la filosofía: poner en cuestión y enfrentar los estados de dominación para ampliar el campo de la libertad. (Kohan, 2003 a: 108)

[... ] y que hay una única educación que vale la pena, la que emancipa sin emancipar. (Kohan, 2003 b: 77).

\section{BIBLIOGRAFÍA}

BORGES, Jorge Luis. Arte poética, España: Crítica, 2001.

CIENFUENTES, Luis M. y otros. Enseñar y aprender filosofía en la educación secundaria. Barcelona: Editorial Hosori. 1997.

HANSON, Norwood Russell. Patrones de descubrimiento. Observación y explicación. Madrid: Alianza Universidad. 1977.

HEGEL, G. W. F. Escritos Pedagógicos. Traducción e Introducción de Arsenio Ginzo, México: F.C.E. 1998.

JASPERS, Karl. La Filosofía, México: F.C.E. 1970.

JOSÉ, Elena Teresa. Textos y pretextos para filosofar. Bs. As: Biblos. 1999.

KANT, I. Crítica de la razón pura. Buenos Aires: Losada. 1965.

KANT, I. "Sobre la enseñanza de la filosofía", en Manía, Revista de la Universidad de Barcelona. Traducción de E. LLedó del Informe de sus clases en la Institución, en el semestre invernal, 1765-1766). n. 1. 1995.

KOHAN, Walter Omar. Infância: Entre Educação e Filosofia, Belo Horizonte: Auténtica. 2003a.

KOHAN, Walter Omar. "Un ejercicio de filosofía de la educación". En: Cuaderno de Pedagogía No 11, Rosario (Argentina) Centro de Estudios en Pedagogía Crítica. $2003 b$.

KONDRÁTOV, A. M. Del sonido al signo. Bs. As: Paidós, 1973. 
OBIOLS, Guillermo A. (diciembre de 1997). Documento sobre el informe de la Consultoría para el proceso de cambio curricular de las Carreras de la Escuela de Filosofía de la Facultad de Humanidades de la Universidad Nacional de Salta.

RANCIÈRE, Jacques. El maestro ignorante. Barcelona: Laertes, 2002.

SALAZAR BONDY, Augusto. Didáctica de la Filosofía. Lima: Ed. Universo1974.

Notas:

(1) Profesora de la Universidad Nacional de Salta, Argentina. Correo electrónico: lilajose@uolsinectis.com.ar

(2) La etimología de la palabra "investigar" fue suministrada por el Servicio de Información sobre la Lengua (SIL), que dirige el Prof. Francisco Jesús Fernández como Director Proyecto Investigación 661 de la Universidad Nacional de Salta, Argentina

(3) Cfr. Obiols, Guillermo, en el libro citado en la bibliografía, pp. 47 a 56.

(4) Las ideas de Foucault sobre "experiencia" y "verdad" fueron extraídas de la Introducción del libro de Kohan, Walter, citado en la bibliografía. 